\title{
Multi-micronutrient supplementation during pregnancy for prevention of maternal anaemia and adverse birth outcomes in a high-altitude area: a prospective cohort study in rural Tibet of China
}

\author{
Yijun Kang ${ }^{1} \dagger$, Shaonong Dang ${ }^{1} \dagger$, Lingxia Zeng ${ }^{1}$, Duolao Wang ${ }^{2}$, Qiang Li $^{1}$, Jianpeng Wang ${ }^{3}$, \\ Luobu Ouzhu ${ }^{4}$ and Hong Yan $^{1 *}$ \\ ${ }^{1}$ School of Public Health, Xi'an Jiaotong University Health Science Center, Xi'an, Shaanxi, 710061, People's Republic of China \\ ${ }^{2}$ Department of Clinical Sciences, Liverpool School of Tropical Medicine, Pembroke Place, Liverpool L3 5QA, UK \\ ${ }^{3}$ The Primary Health and Maternal and Child Health Division of Health Bureau of Tibet Autonomous Region, Lhasa, Tibet \\ Autonomous Region, 850000, People's Republic of China \\ ${ }^{4}$ School of Medicine, Tibet University, Lhasa, Tibet Autonomous Region, 850000, People's Republic of China
}

(Submitted 20 December 2016 - Final revision received 27 July 2017 - Accepted 1 August 2017)

\section{Abstract}

Anaemia during pregnancy, characterised by $\mathrm{Hb}<110 \mathrm{~g} / \mathrm{l}$, is a specific risk factor for adverse maternal and perinatal outcomes in developing countries. The objective of this study was to determine the effectiveness of daily antenatal supplementation with multiple micronutrients (MMN) compared with folic acid (FA) on the occurrence of anaemia among pregnant women and their infants' health in a high-altitude area. A prospective cohort study was carried out in two rural counties in Tibet from 2007 to 2012. A total of 1149 eligible pregnant women were allocated daily supplementation with FA in one county and MMN containing a recommended allowance of twenty-three vitamins and minerals in another county starting $\leq 24$ weeks of gestation and continuing until delivery. Compared with the FA group, prenatal supplementation with MMN was significantly associated with reduced odds of anaemia in the third trimester. This was demonstrated in the primary outcome, with an adjusted OR (AOR) of $0.63 ; 95 \%$ CI $0.45,0.88$ and $P=0.007$ and also reduced odds of preterm delivery (AOR: $0.31 ; 95 \%$ CI $0.15,0.61$; $P=0.001$ ). There was no difference between MMN and FA groups in mean birth weight (adjusted mean difference: $36.78 ; 95 \%$ CI -19.42 , 92.98 g; $P=0.200$ ), whereas MMN supplementation significantly reduced the odds of low-birth weight (LBW) babies (AOR: 0.58 ; $95 \%$ CI 0.36 , 0.91; $P=0.019$ ). In conclusion, the antenatal MMN supplementation in rural Tibet is associated with a reduction of maternal anaemia in the third trimester, and may potentially decrease the risk of preterm delivery and LBW babies.

Key words: Multiple micronutrient supplementation: Anaemia: Low birth weight: Preterm delivery: Rural Tibet

Anaemia during pregnancy is a considerable health problem around the world and Fe deficiency is the most common cause of anaemia ${ }^{(1,2)}$. Globally, the prevalence of anaemia in pregnant women has decreased since 1995 , but in $201138 \%$ of pregnant women still suffered from anaemia ${ }^{(1)}$. In 2012, the prevalence of anaemia during pregnancy in China was $17 \cdot 2 \%{ }^{(3)}$; however, the prevalence of anaemia among pregnant women strongly varies by region and women's gestational age. The China National Nutrition and Health Survey showed that the prevalence of anaemia was $20 \%$ among pregnant women in poor rural areas $^{(4)}$. A prospective cohort study in south China indicated that about $70 \%$ of pregnant women had Fe-deficiency anaemia ${ }^{(5)}$. There is very limited information on anaemia status of Tibetan pregnant women. Our previous hospital-based study in Lhasa showed that Tibetan pregnant women had lower Hb levels than
non-Tibetan women throughout the entire pregnancy, averaging $126.6 \mathrm{~g} / \mathrm{l}$ for Tibetans and $134.6 \mathrm{~g} / \mathrm{l}$ for non-Tibetans ${ }^{(6)}$. The percentage of women with $\mathrm{Hb}<110 \mathrm{~g} / \mathrm{l}$ was $18.5 \%$ before correction for altitudes and $71.2 \%$ after correction for altitudes $^{(7)}$. Another population-based study showed that the prevalence of anaemia in pregnant women during the first, second and third trimester in rural Tibet was $79 \cdot 5,86 \cdot 1$ and $87 \cdot 3 \%$, respectively ${ }^{(8)}$. These findings suggested that Tibetan women were more likely to be anaemic during pregnancy, compared with those in other regions in China.

Anaemia during pregnancy is associated with adverse birth outcomes and has long-term effects. Anaemia in the third trimester of pregnancy increases the incidence of low birth weight (LBW) and preterm birth ${ }^{(5,9)}$. Hb reduction during pregnancy is associated with small for gestational age (SGA)

Abbreviations: AOR, adjusted OR; FA, folic acid; GEE, generalised estimating equation; LBW, low birth weight; MMN, multiple micronutrients.

* Corresponding author: Dr H. Yan, email xjtu_yh.2014@163.com

$\dagger$ These authors contributed equally to this work. 
births, placental weight and placental ratio ${ }^{(10-12)}$. LBW babies are at higher risks of morbidity and mortality, and of adverse physical development in childhood, compared with normal birth weight babies $^{(13,14)}$. Anaemia during pregnancy might have long-term adverse effects on younger children, and a study indicated that Fe-deficiency anaemia in the third trimester was associated with worse mental development of rural Chinese young children ${ }^{(15)}$.

Tibet is located in the Qinghai-Tibet plateau, where more than $45 \%$ of areas are above the altitude of $4000 \mathrm{~m}^{(16)}$. Hypoxia at high altitude significantly increases $\mathrm{Hb}^{(17)}$. However, the relationship between high altitude and $\mathrm{Hb}$ is less clear in those adapted to high altitudes, such as Tibetans ${ }^{(7,18,19)}$. Thus, anaemic status and its adverse effects during pregnancy among Tibetans require further investigation. Previous cross-sectional surveys showed that the nutritional status of women and children in Tibet was worse than that in any other regions in China ${ }^{(6-8,20,21)}$. The LBW rate was $10.5 \%$ in rural Tibet, higher than that in other regions in China $(4.6 \%)^{(22,23)}$. However, there is a lack of follow-up studies on anaemia and LBW among the pregnant Tibetan women.

Deficiencies in micronutrients during pregnancy are common in developing countries and have been associated with maternal anaemia and adverse birth outcomes. These deficiencies may be exacerbated during pregnancy because of increased requirements for the growing fetus, placenta and maternal tissues $^{(24)}$. Globally, anaemia due to Fe deficiency is one of the most prevalent micronutrient deficiencies. Therefore, preventive measures are needed to reduce and control anaemia during pregnancy. Some preventative measures during pregnancy that are recommended include the fortification of principal foods with $\mathrm{Fe}$, the increase of health and nutritional awareness and the improvement in sanitation. However, the most direct way to improve Fe status and reduce the occurrence of anaemia is suggested to be antenatal supplementation with Fe-containing micronutrients ${ }^{(15,24,25)}$. A systematic review and meta-analysis including forty-eight randomised trials (twentyseven in high-income countries and twenty-one in low- or middle-income countries; the dosage of Fe ranged from 10 to $240 \mathrm{mg}$ daily and duration of supplementation varied from 7 to 30 weeks during pregnancy) and forty-four cohort studies (twenty-two each from high-income and low- or middle-income countries, respectively) was conducted to assess prenatal $\mathrm{Fe}$ supplements ${ }^{(26)}$. The review showed that Fe use during pregnancy increased maternal mean $\mathrm{Hb}$ level by $4.59 \mathrm{~g} / \mathrm{l}$ and in turn reduced the risk of anaemia, Fe deficiency, Fe deficiency anaemia and LBW. These effects were particularly strong among pregnant women with anaemic status at baseline or those from countries with low or middle income ${ }^{(26)}$. Thus, this study suggested that the effects of micronutrient supplements containing $\mathrm{Fe}$ during pregnancy might vary with region, population and anaemic status at the beginning of intervention.

Tibet is a very specific setting characterised with high altitudes and hypoxia, and the effects of Fe supplementation during pregnancy at high altitudes on maternal $\mathrm{Hb}$, anaemia and birth outcomes remain unknown. Therefore, we conducted a prospective cohort study in rural Tibet to assess the positive impacts of multiple micronutrients (MMN) supplementation containing $\mathrm{Fe}$ during pregnancy on maternal anaemia and adverse birth outcomes such as LBW and preterm delivery.

\section{Methods}

\section{Study design and population}

A prospective cohort study was conducted in two rural counties (Dazi and Qushui), in Lhasa, located in the middle of Tibet. The mean altitude of the two counties is $3706 \mathrm{~m}$ above sea level. Lhasa area is one of the main habitats of Tibetans.

This study was approved by the Ethics Committee in Medical Research, Xi'an Jiaotong University (no 20070712), and registered in the Chinese Clinical Trial Registry (Registration no.: ChiCTR-PNRC-09000597).

The study sample consisted of the pregnant women in the two counties who met the study selection criteria (Fig. 1). The women having $\leq 24$ weeks of gestation were invited to participate in the study and had to provide signed informed consent. The women were excluded from the study if they already took $\mathrm{Fe}$, folic acid (FA) or other micronutrient supplements for more than two weeks, or had other serious illnesses. The eligible pregnant women from Dazi took MMN, whereas those from Qushui took FA. Randomisation was not implemented for the reasons of cultural and religious sensitivity in the study population. FA is the standard prenatal supplementation recommended by the National Health and Family Planning Commission of China, so FA was used as the control ${ }^{(27)}$. All participants were required to take the supplements from enrolment to delivery. The sample size considerations were based on a survey in rural Lhasa areas. The anaemia prevalence of pregnant women in the third trimester in rural Lhasa is $75.9 \%$ (adjusted by altitude) $^{(6)}$. Taking MMN supplements during pregnancy was assumed to reduce anaemia prevalence by $11 \%$ compared with $\mathrm{FA}^{(28)}$. Using $\alpha=0.05$ and power $=80 \%, 920$ subjects were required. A total of 1100 pregnant women were required after accounting for about $15 \%$ dropout/loss to follow-up.

\section{Enrolment and pregnancy surveillance procedures}

At the outset of this study, the trained village doctors and social workers conducted a survey of all women of reproductive age living in the village to identify those who were likely to become pregnant. The village doctors visited these women every month and asked them the date of their last menstrual period (LMP). The women whose periods were delayed by more than $5 \mathrm{~d}$ were given urine pregnancy tests, and confirmed pregnancies were reported to the maternal and child health care $(\mathrm{MCH})$ workers in the township hospitals of the studied counties. The informed consent was obtained from all participants.

Newly identified pregnant women were interviewed to record their socio-demographic status and menstrual, reproductive and medical histories. Recruited pregnant women received three free antenatal care checks at enrolment, 28 and 32 weeks of gestation, respectively, at which they were given physical examination and asked about pregnancy complications. All the information collected during the pregnancy until the sixth week after delivery follow-up visit was recorded in the Pregnancy Care Record Book, which served as both a clinical record and a data capture instrument. 


\section{Micronutrient supplements}

In this study, two kinds of supplements were used: MMN and FA. The MMN supplement (Materna/tablet; Wyeth Pharmaceutical Co., Ltd) consisted of the following components (per tablet): $450 \mu \mathrm{g}$ of vitamin A, $900 \mu \mathrm{g}$ of $\beta$-carotene, $6 \cdot 25 \mu \mathrm{g}$ of vitamin $\mathrm{D}, 30 \mathrm{mg}$ of vitamin $\mathrm{E}, 3 \mathrm{mg}$ of vitamin $\mathrm{B}_{1}, 3.4 \mathrm{mg}$ of vitamin $B_{2}, 10 \mathrm{mg}$ of vitamin $B_{6}, 12 \mu \mathrm{g}$ of vitamin $B_{12}, 100 \mathrm{mg}$ of vitamin C, $30 \mathrm{mcg}$ of biotin, $1 \mathrm{mg}$ of FA, $20 \mathrm{mg}$ of nicotinamide, $10 \mathrm{mg}$ of pantothenic acid, $150 \mu \mathrm{g}$ of iodine, $25 \mu \mathrm{g}$ of Mo, $250 \mathrm{mg}$ of $\mathrm{Ca}, 25 \mathrm{mg}$ of $\mathrm{Zn}, 60 \mathrm{mg}$ of $\mathrm{Fe}, 2.0 \mathrm{mg}$ of $\mathrm{Cu}, 25.0 \mu \mathrm{g}$ of $\mathrm{Cr}, 5 \mathrm{mg}$ of $\mathrm{Mn}, 50 \mathrm{mg}$ of $\mathrm{Mg}$ and $25.0 \mu \mathrm{g}$ of Se. The folate-only supplement (Scrianen/tablet; Peking University Pharmaceutical Co., Ltd) contained $0.4 \mathrm{mg}$ of FA/tablet. All micronutrient supplements were commercially available at the time of the study.

At enrolment, each woman received thirty tablets with the instructions to take one tablet daily, preferably after meals and at the same time every day. Either the township $\mathrm{MCH}$ workers or the village doctors visited the women every month to provide supplements and to retrieve the used empty bottles and record the number of remaining tablets.

\section{Data collection}

Maternal $\mathrm{Hb}$ during pregnancy was tested from the capillary blood collected at enrolment ( $\leq 24$ weeks of gestation), 28 and 32 weeks of gestation, respectively. HemoCue portable spectrophotometers were used to assay $\mathrm{Hb}$ levels. The method of American Centers for Disease Control and Prevention (CDC) was used in this study to adjust Hb levels based on the altitude for estimating the anaemia prevalence. The adjusted formula is expressed as $\Delta \mathrm{Hb}=-0.032 \times($ Alt $\times 3 \cdot 3)+0.022 \times(\text { Alt } \times 3.3)^{2}$, where Alt refers to the altitude $(\mathrm{km})$ of the village where the participant lived ${ }^{(17)}$. Participants whose adjusted $\mathrm{Hb}$ level was $<110 \mathrm{~g} /$ l were considered anaemic ${ }^{(25,29)}$.

Birth weight and length were measured by the hospital nurses within $1 \mathrm{~h}$ of a child's delivery. Birth weight was measured using an electronic scale (Type BD-585; TANITA Corporation, Ltd) with precision to the nearest $10 \mathrm{~g}$. For home births ( $11 \%$ of all births), the township $\mathrm{MCH}$ workers visited the women at home within $72 \mathrm{~h}$ of delivery to measure the birth anthropometry and collect the delivery information. LBW was defined as birth weight $<2500 \mathrm{~g}^{(30)}$. Birth length was measured to the nearest $1 \mathrm{~mm}$ using a portable measuring board with a fixed head piece.

Gestational age at birth was calculated as completed days based on the 1st day of the LMP, which was obtained from the baseline interview. Spontaneous abortion was defined as the spontaneous end of confirmed pregnancy before 28 weeks of gestational age. Preterm delivery was defined as delivery at $<37$ weeks of gestational age. A stillbirth is defined as the spontaneous death of a fetus after 28 weeks of gestation but before delivery of the baby's head. Neonatal deaths were defined as deaths amongst live-born infants occurring within $28 \mathrm{~d}$ of delivery. Early neonatal death was defined as the death within $7 \mathrm{~d}$ after birth. Late neonatal death was referred to as death between 8 and $28 \mathrm{~d}$ after birth. We also reported perinatal mortality, which included both stillbirths and early neonatal deaths.

\section{Statistical analysis}

A wealth index was constructed from an inventory of six household assets (including the following family belongings: motorcycles, automobiles, cattle, goats, chickens or ducks, televisions) using a principal component analysis method ${ }^{(31)}$. The compliance rate was determined by dividing the number of actual supplements consumed by the number of supplements expected to be consumed. In the analysis, we only included singleton pregnancy to avoid the possible contamination effect on anaemia and birth outcomes because the multiple pregnancies are more vulnerable to those outcomes.

To address the possible imbalance in the participants' characteristics between the two treatment groups owing to the nonrandomised design of this study, a propensity score approach was used. We calculated the propensity score (a predicted probability of woman being in Dazi county) for each woman using a logistic regression model based on fourteen prespecified baseline characteristics (except $\mathrm{Hb}$ level adjusted by altitude) as listed in Table 2 and used this score as a control variable in the covariate-adjusted analysis of the outcome variables $^{(32)}$.

The primary outcome was analysed using a generalised estimating equation (GEE) model with treatment, time at measurement, interaction between treatment and time as fixed effects, and the propensity score and $\mathrm{Hb}$ level (adjusted by altitude) at baseline as covariates. OR, adjusted OR and its $95 \%$ CI for having anaemia during prenatal care between two treatment groups were derived from the GEE model. Other outcome variables were analysed similarly using a GEE model. For the GEE model analysis of the continuous outcome such as $\mathrm{Hb}$ level, weeks of gestation at birth and birth weight, normal distribution and identity link functions were used. For the GEE analysis of the binary outcomes such as anaemia, preterm delivery, LBW and mortality outcomes, binomial distribution and logit link functions were used. An exchangeable covariance structure was used for all GEE models. In addition, the twosample $t$ test or Wilcoxon's rank test was used to assess statistical differences in continuous variables between the two supplementation groups. $P<0.05$ was considered statistically significant. All analyses were performed using SAS version 9.3 (SAS Institute).

\section{Results}

\section{Study sample and characteristics}

Fig. 1 shows the flow chart of participants in this study. The enrolment began on 26 October 2007, and continued until 4 May 2011. The last baby was born on 12 December 2011, and all follow-up stopped on 6 March 2012. Over a 4.5-year period, there were 2821 confirmed pregnant women who lived in the two rural counties of Qushui and Dazi in Lhasa. Among them, 568 pregnant women refused to participate and 1104 women were excluded from the study as per the exclusion criteria. Thus, in total, 1149 pregnant women were enrolled in the study. There were 1013 singleton pregnant women in the third trimester available for analysis. The birth weights were not 


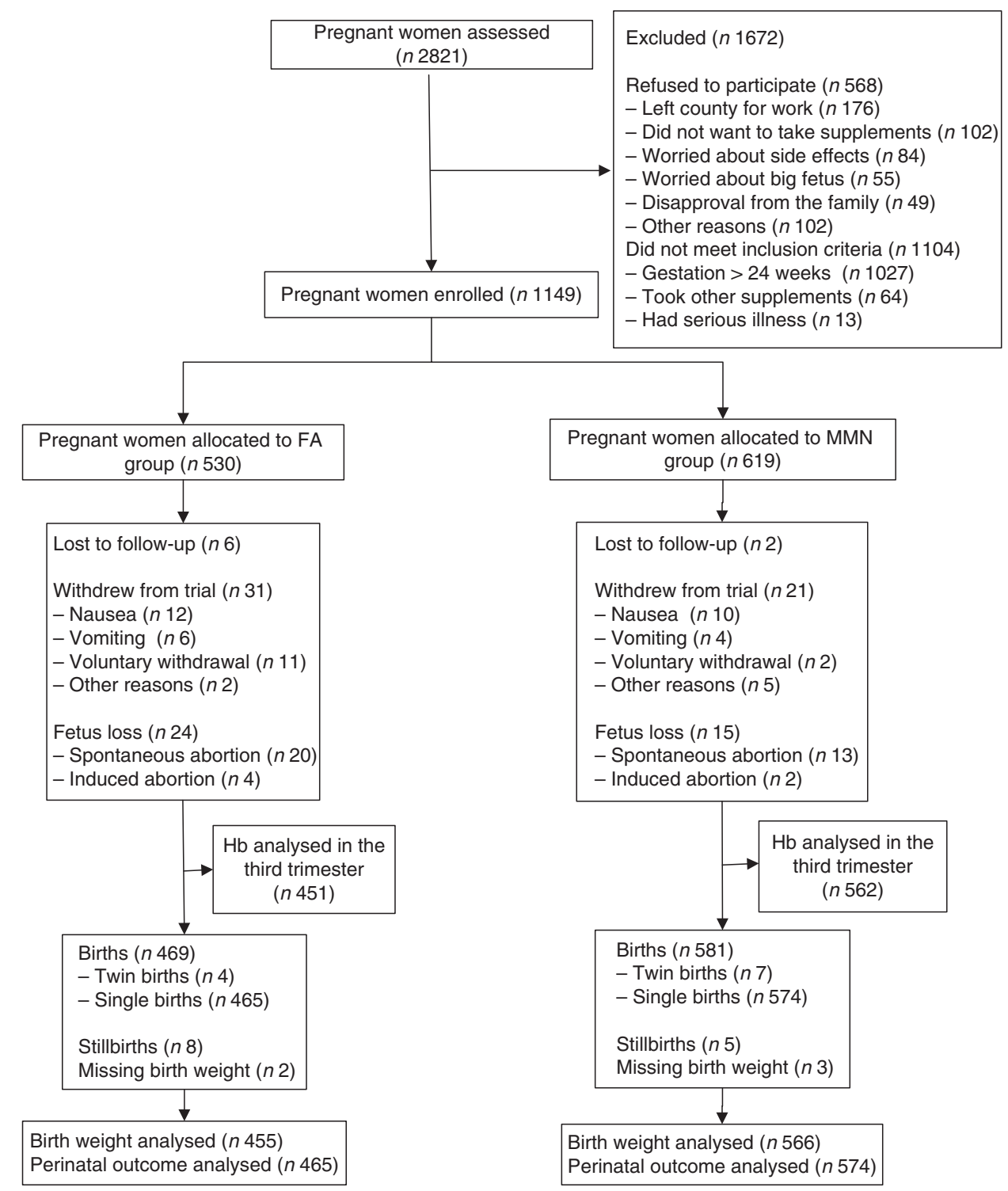

Fig. 1. Flow chart depicting participant selection process. FA, folic acid; $M M N$, multiple micronutrients.

measured for thirteen stillbirths and the birth weight data for five live births were missed. Therefore, the birth weights of 1021 singleton live births were available for analysis.

The two counties, Qushui and Dazi, are almost identical in terms of geographical features and social status, such as population size, economic situation, medical service level and the number of women of childbearing age (Table 1). Characteristics of the pregnant women at enrolment are shown in Table 2. All participants were Tibetan. Most were educated, but only $45.6 \%$ had completed an elementary school education. In all, $17.4 \%$ had low BMI (BMI $\left.<18.5 \mathrm{~kg} / \mathrm{m}^{2}\right)$ and 499 participants $(43.4 \%)$ were in the first pregnancies.

\section{Compliance with supplementation}

The supplementation began, on average, at $15 \cdot 0$ (SD 5.0) weeks of gestation for both groups. The mean dosage number of
Table 1. Baseline status of two counties in 2006

\begin{tabular}{lcc}
\hline Variables & Qushui county & Dazi county \\
\hline Area $\left(\mathrm{km}^{2}\right)$ & 1624 & 1373 \\
No. of villages & 17 & 21 \\
Altitude $(\mathrm{m})$ & & \\
$\quad$ Mean* & 3634 & 3768 \\
SD & 47 & 70 \\
Population & 30968 & 27196 \\
Per capita GDP (RMB yuan) & 9800 & 10000 \\
Rate of cooperative medical service (\%) & 100 & 100 \\
No. of women of childbearing age & 8716 & 8239 \\
Birth rate (\%) & 14.5 & $15 \cdot 5$ \\
No. of village clinics & 11 & 18 \\
No. of township hospitals & 5 & 5 \\
No. of county hospitals & 1 & 1 \\
No. of MCH staff & 9 & 8 \\
\hline
\end{tabular}

$\mathrm{MCH}$, maternal and child health care.

${ }^{*} t$ Test was used to examine statistical difference in means, $t=38.80$, $P<0.001$. 
Table 2. Baseline household and participant characteristics at enrolment (Mean values and standard deviations; medians and interquartile ranges (IQR))

\begin{tabular}{|c|c|c|c|c|c|}
\hline \multirow[b]{2}{*}{ Variables } & \multicolumn{2}{|c|}{ FA $(n$ 465) } & \multicolumn{2}{|c|}{ MMN ( $n$ 574) } & \multirow[b]{2}{*}{$P^{\star}$} \\
\hline & Mean & SD & Mean & SD & \\
\hline \multicolumn{6}{|l|}{ Socio-demographics } \\
\hline Number of household members & $5 \cdot 0$ & 1.9 & 4.6 & 1.7 & $<0.001$ \\
\hline Per capita income per year (RMB) & & & & & 0.004 \\
\hline Median & \multicolumn{2}{|c|}{1500} & \multicolumn{2}{|c|}{1667} & \\
\hline IQR & \multicolumn{2}{|c|}{1400} & \multicolumn{2}{|c|}{1747} & \\
\hline Household wealth index & & & & & 0.171 \\
\hline Median & \multirow{2}{*}{\multicolumn{2}{|c|}{$\begin{array}{l}0.42 \\
1.33\end{array}$}} & \multicolumn{2}{|c|}{0.42} & \\
\hline IQR & & & & & \\
\hline Father's age (years) & $27 \cdot 7$ & 4.8 & $27 \cdot 6$ & $5 \cdot 0$ & 0.655 \\
\hline Mother's age (years) & $25 \cdot 6$ & 4.8 & $25 \cdot 4$ & 4.4 & 0.417 \\
\hline \multicolumn{6}{|l|}{ Education } \\
\hline Father's education (years) & $5 \cdot 7$ & 3.7 & $5 \cdot 1$ & 3.4 & 0.014 \\
\hline Mother's education (years) & $5 \cdot 2$ & 3.8 & 4.8 & 3.5 & 0.095 \\
\hline \multicolumn{6}{|l|}{ Reproductive history } \\
\hline Menarcheal age (years) & $15 \cdot 8$ & 1.5 & $15 \cdot 2$ & 1.5 & $<0.001$ \\
\hline Interval of menstruation (d) & $29 \cdot 0$ & $2 \cdot 8$ & $28 \cdot 1$ & $2 \cdot 2$ & $<0.001$ \\
\hline Continuous days of menstruation & 3.8 & 1.0 & 3.8 & 1.0 & 0.568 \\
\hline Parity & 1.8 & 0.9 & 1.8 & 0.8 & 0.100 \\
\hline Gestational age (weeks) & $15 \cdot 0$ & $5 \cdot 0$ & $15 \cdot 0$ & $5 \cdot 0$ & 0.854 \\
\hline \multicolumn{6}{|l|}{ Anthropometric measurements } \\
\hline Mother BMI $\left(\mathrm{kg} / \mathrm{m}^{2}\right)$ & $20 \cdot 8$ & $2 \cdot 6$ & 20.7 & $2 \cdot 2$ & 0.604 \\
\hline $\mathrm{Hb}(\mathrm{g} / \mathrm{l})$ & $126 \cdot 0$ & 21.4 & 128.6 & $21 \cdot 0$ & 0.049 \\
\hline $\mathrm{Hb}$ (adjusted by altitude) (g/l) & $98 \cdot 2$ & $21 \cdot 4$ & 98.5 & $21 \cdot 2$ & 0.795 \\
\hline
\end{tabular}

Table 3. Number of doses and compliance of supplements consumed by supplementation groups* (Numbers and percentages; mean values and standard deviations)

\begin{tabular}{|c|c|c|c|c|c|}
\hline \multirow[b]{2}{*}{ Dose and compliance } & \multicolumn{2}{|c|}{$\mathrm{FA}(n 465)$} & \multicolumn{2}{|c|}{ MMN $(n$ 574) } & \multirow[b]{2}{*}{$P+$} \\
\hline & $n$ & $\%$ & $n$ & $\%$ & \\
\hline \multicolumn{6}{|l|}{ Supplements (capsules) } \\
\hline \multicolumn{6}{|c|}{ Consumed distribution } \\
\hline$<90$ & 17 & 3.7 & 30 & $5 \cdot 2$ & \\
\hline $90-120$ & 64 & $13 \cdot 8$ & 91 & $15 \cdot 9$ & \\
\hline $121-180$ & 273 & $58 \cdot 7$ & 322 & $56 \cdot 1$ & \\
\hline$>180$ & 111 & 23.9 & 131 & $22 \cdot 8$ & \\
\hline Consumed & & & & & 0.141 \\
\hline Mean & \multicolumn{2}{|c|}{151} & \multicolumn{2}{|c|}{148} & \\
\hline SD & \multicolumn{2}{|c|}{35} & \multicolumn{2}{|c|}{37} & \\
\hline \multicolumn{6}{|l|}{ Compliance distribution } \\
\hline $0-50$ & 2 & 0.4 & 8 & 1.4 & \\
\hline $50 \cdot 1-75$ & 41 & 8.8 & 106 & $18 \cdot 5$ & \\
\hline $75 \cdot 1-100$ & 422 & $90 \cdot 8$ & 460 & $80 \cdot 1$ & \\
\hline Compliance & & & & & $<0.001$ \\
\hline Mean & \multicolumn{2}{|c|}{$91 \cdot 3$} & \multicolumn{2}{|c|}{$85 \cdot 7$} & \\
\hline SD & \multicolumn{2}{|c|}{$10 \cdot 2$} & \multicolumn{2}{|c|}{$12 \cdot 9$} & \\
\hline
\end{tabular}

FA, folic acid; MMN, multiple micronutrients.

* Compliance calculated by number of actual supplements consumed divided by number of supplements expected to be consumed. $\dagger t$ Test was used to examine statistical differences in means.

supplements consumed per woman during pregnancy was 151 (sD 35) in the FA group and 148 (SD 37) in the MMN group $(P=0 \cdot 141)$. The compliance with supplementation during pregnancy was $88.2 \%(91.3 \%$ in the FA group and $85.7 \%$ in the MMN group; $P<0.001$ ) (Table 3 ). There were a few self-reported side effects, such as nausea, vomiting, headache and dizziness. The prevalence of such side effects was below $2.3 \%$ and comparable between the two treatment groups.

\section{Comparison of maternal anaemia between the supplementation groups}

Blood Hb samples were available for 1037 women at enrolment, 1031 in the second prenatal care and 1013 in the third prenatal care. The blood samples were taken at a mean gestation of $15 \cdot 0$ (sD 5.0), 28.3 (SD 1.1) and 32.2 (SD 1.2) weeks for the first, second and third prenatal care, respectively. There was a significant 
Table 4. Comparison of anaemia and $\mathrm{Hb}(\mathrm{g} / \mathrm{l})$ of Tibetan pregnant women between supplementation groups (Mean values and standard deviations; odds ratios, adjusted odds ratios (AOR) or differences and $95 \%$ confidence intervals)

\begin{tabular}{|c|c|c|c|c|c|c|c|c|c|}
\hline & \multirow[b]{2}{*}{$n^{*}$} & \multirow[b]{2}{*}{ Mean } & \multirow[b]{2}{*}{ SD } & \multicolumn{6}{|c|}{ MMN v. FA } \\
\hline & & & & OR or difference & $95 \% \mathrm{Cl}$ & $P$ & AOR or difference $†$ & $95 \% \mathrm{Cl}$ & $P$ \\
\hline \multicolumn{10}{|c|}{ Anaemia (adjusted by altitude)‡ } \\
\hline \multicolumn{10}{|c|}{ The 28.3 weeks of gestation (\%) } \\
\hline FA & 461 & & & & & & & & \\
\hline MMN & 570 & & & 0.81 & $0.60,1.07$ & 0.140 & 0.69 & $0.50,0.96$ & 0.028 \\
\hline \multicolumn{10}{|c|}{ The 32.2 weeks of gestation (\%) } \\
\hline FA & 451 & & & & & & & & \\
\hline MMN & 562 & & & 0.74 & $0.56,0.99$ & 0.039 & 0.63 & $0.45,0.88$ & 0.007 \\
\hline \multicolumn{10}{|c|}{$\mathrm{Hb}$ (adjusted by altitude) } \\
\hline \multicolumn{10}{|c|}{ The 28.3 weeks of gestation } \\
\hline FA & 461 & $95 \cdot 6$ & $18 \cdot 8$ & & & & & & \\
\hline MMN & 570 & $99 \cdot 3$ & $18 \cdot 2$ & $3 \cdot 24$ & $0.88,5 \cdot 60$ & 0.007 & 4.07 & $2 \cdot 00,6 \cdot 15$ & 0.001 \\
\hline \multicolumn{10}{|c|}{ The $32 \cdot 2$ weeks of gestation } \\
\hline FA & 451 & 95.5 & $18 \cdot 2$ & & & & & & \\
\hline MMN & 562 & $100 \cdot 3$ & $18 \cdot 1$ & 4.31 & $1.69,6.94$ & 0.001 & $5 \cdot 18$ & $2 \cdot 98,7.37$ & $<0.001$ \\
\hline
\end{tabular}

FA, folic acid; MMN, multiple micronutrients.

* Data missing on the 28.3 weeks of gestation for 8 women, and the 32.2 weeks of gestation for twenty-six women.

$\dagger$ Adjusted for the propensity scores and $\mathrm{Hb}$ levels (adjusted by altitude) at enrolment in generalised estimating equation model.

$\ddagger$ Anaemia defined as $\mathrm{Hb}<110 \mathrm{~g} / \mathrm{l}$.

Table 5. Comparison of outcomes of child health between supplementation groups

(Mean values and standard deviations; odds ratios, adjusted odds ratios (AOR) or differences and $95 \%$ confidence intervals)

\begin{tabular}{|c|c|c|c|c|c|c|c|c|c|c|c|c|}
\hline \multirow[b]{2}{*}{ Indicators } & \multicolumn{3}{|c|}{ FA } & \multicolumn{3}{|c|}{ MMN } & \multicolumn{6}{|c|}{ MMN v. FA } \\
\hline & $\begin{array}{l}\text { No. in } \\
\text { group }\end{array}$ & Mean & SD & $\begin{array}{l}\text { No. in } \\
\text { group }\end{array}$ & Mean & SD & $\begin{array}{l}\text { Difference } \\
\text { or OR }\end{array}$ & $95 \% \mathrm{Cl}$ & $P$ & $\begin{array}{c}\text { Adjusted } \\
\text { difference or } \\
\text { AOR }^{*}\end{array}$ & $95 \% \mathrm{Cl}$ & $P$ \\
\hline \multicolumn{13}{|l|}{ Continuous outcomes } \\
\hline Birth weight $(\mathrm{g}) \dagger$ & 455 & 2981.9 & $475 \cdot 7$ & 566 & $3006 \cdot 9$ & 387.2 & $26 \cdot 50$ & $-26.50,79.49$ & 0.327 & 36.78 & $-19.42,92.98$ & 0.200 \\
\hline Birth length $(\mathrm{cm}) \dagger$ & 433 & 48.4 & 3.6 & 562 & 48.4 & $3 \cdot 2$ & 0.02 & $-0.41,0.44$ & 0.930 & 0.02 & $-0.43,0.47$ & 0.939 \\
\hline Gestation at birth (weeks) $\ddagger$ & 465 & 39.1 & 1.9 & 574 & 39.6 & 1.7 & 0.49 & $0.27,0.71$ & $<0.001$ & 0.56 & $0.33,0.79$ & $<0.001$ \\
\hline \multicolumn{13}{|l|}{ Binary outcomes (\%) } \\
\hline $\begin{array}{l}\text { Low birth weight } \\
(<2500 \mathrm{~g}) \dagger\end{array}$ & 455 & $9 \cdot 5$ & & 566 & 6.5 & & 0.64 & $0.41,1.00$ & 0.052 & 0.58 & $0.36,0.91$ & 0.019 \\
\hline Preterm‡ & 465 & $7 \cdot$ & & 574 & 2.3 & & 0.30 & $0.16,0.58$ & $<0.001$ & 0.31 & $0.15,0.61$ & 0.001 \\
\hline
\end{tabular}

FA, folic acid; MMN, multiple micronutrients.

* Adjusted for the propensity scores in generalised estimating equation model.

$\dagger$ Data analysis only includes single live births.

‡ Data analysis only includes single births.

increase in the $\mathrm{Hb}$ with MMN supplement compared with FA supplement in the third trimester (adjusted mean difference: 5.18; $95 \%$ CI $2.98,7.37 \mathrm{~g} / 1 ; P<0 \cdot 001)$, resulting in significant reduced odds of anaemia in the third trimester for these women (AOR: 0.63; $95 \%$ CI 0.45, 0.88; $P=0 \cdot 007$ ) (Table 4).

\section{Comparison of birth outcomes between the supplementation groups}

Birth weight and length samples were available for 1021 and 995 single live births, respectively. After the supplementation, the mean birth weight was 2981.9 (SD 475.7) $\mathrm{g}$ in the FA group and 3006.9 (SD 387.2) $\mathrm{g}$ in the MMN group (adjusted mean difference: $36 \cdot 78 ; 95 \% \mathrm{CI}-19 \cdot 42,92 \cdot 98 \mathrm{~g} ; P=0 \cdot 200$ ). The effect of supplementation on birth length was not statistically significant (adjusted mean difference: $0.02 ; 95 \%$ CI $-0.43,0.47 \mathrm{~cm}$; $P=0.939)$. MMN resulted in a significantly longer gestation compared with that of FA (adjusted mean difference: 0.56;95\%
CI $0.33,0.79$ weeks; $P<0.001)$. There were significant decreases in the odds of LBW and preterm delivery in the MMN group compared with the FA group (AOR: 0.58 ; $95 \%$ CI $0.36,0.91$; $P=0.019$; AOR: $0 \cdot 31 ; 95 \%$ CI $0 \cdot 15,0 \cdot 61 ; P=0.001$; respectively) (Table 5).

MMN supplementation was associated with decreased perinatal mortality compared with FA supplementation (AOR: 0.46; $95 \%$ CI $0.23,0.94 ; P=0.034)$, but it showed no effects on the rates of neonatal mortality, early neonatal mortality and stillbirth, although more stillbirths and neonatal death mortality were reported in the FA group (Table 6).

\section{Discussion}

To the best of our knowledge, this is the first intervention study on prenatal MMN supplementation on maternal anaemia and adverse birth outcomes among Tibetan women living at higher altitudes. The present study found that the prenatal MMN 
Table 6. Comparison of neonatal deaths and fetal loss between supplementation groups (Odds ratios, adjusted odds ratios (AOR) and $95 \%$ confidence intervals)

\begin{tabular}{|c|c|c|c|c|c|c|c|c|c|c|}
\hline \multirow[b]{2}{*}{ Indicators } & \multicolumn{2}{|l|}{ FA } & \multicolumn{2}{|c|}{ MMN } & \multicolumn{6}{|c|}{ Comparing MMN with FA } \\
\hline & No. in group & $\%$ & No. in group & $\%$ & OR & $95 \% \mathrm{Cl}$ & $P$ & $\mathrm{AOR}^{*}$ & $95 \% \mathrm{Cl}$ & $P$ \\
\hline Neonatal death & 457 & $26 \cdot 26$ & 569 & $19 \cdot 33$ & 0.73 & $0.32,1.67$ & 0.458 & 0.54 & $0.24,1.23$ & 0.144 \\
\hline Early neonatal death & 457 & $24 \cdot 07$ & 569 & $15 \cdot 82$ & 0.65 & $0.27,1.59$ & 0.345 & 0.47 & $0 \cdot 20,1 \cdot 11$ & 0.086 \\
\hline Stillbirth & 465 & $17 \cdot 20$ & 574 & $8 \cdot 71$ & 0.50 & $0.16,1.55$ & 0.230 & 0.46 & $0.14,1.54$ & 0.209 \\
\hline Perinatal death & 465 & $40 \cdot 86$ & 574 & 24.39 & 0.59 & $0.29,1.18$ & 0.136 & 0.46 & $0.23,0.94$ & 0.034 \\
\hline
\end{tabular}

FA, folic acid; MMN, multiple micronutrients.

* Adjusted for the propensity scores in generalised estimating equation model.

supplementation increased $\mathrm{Hb}$ levels and reduced anaemia significantly during the third trimester compared with the FA supplementation. Furthermore, MMN tended to increase gestational duration and decrease the risks of LBW and preterm delivery. This study provides some important evidence indicating that the MMN supplementation should be required in formulating nutrition policy on nutrient supplementation for pregnant women at high-altitude areas.

\section{Improvement of maternal anaemia}

In the present study, MMN or FA was administered to two groups of Tibetan pregnant women. The Hb of the MMN group increased during the third trimester by $5 \cdot 18 \mathrm{~g} / \mathrm{l}$ at 32 weeks of gestation, significantly higher compared with the FA group. Accordingly, the supplementation with MMN decreased the risk of maternal anaemia by $37 \%$ after controlling for the potential confounders compared with FA. These results further confirmed the possibility that the supplementation with Fe-containing micronutrients during pregnancy improved maternal anaemia, which would benefit both the mother and the child ${ }^{(26)}$. However, the supplementation with Fe-containing micronutrients for pregnant Tibetan women might be even more necessary because rural Tibetan mothers had fairly monotonous dietary patterns with traditional Tibetan foods ${ }^{(20)}$. As anaemia prevalence was high among pregnant women in rural Tibet ${ }^{(6,8)}$, the routine supplementation of Fe-containing micronutrients during pregnancy should be included in antenatal care among Tibetan women at higher altitudes.

A cluster-randomised trial from rural western China showed that the supplementation with MMN during pregnancy could decrease the risk of maternal anaemia by $28 \%$ compared with supplementation with $\mathrm{FA}^{(33)}$. MMN in the present study decreased the risk of maternal anaemia by $37 \%$, which was a significant improvement. Although the observed difference should not be overstated because of the non-randomised nature of this study, the difference might also be due to higher dose of Fe in MMN supplements of the present study $(60 \mathrm{mg} \mathrm{Fe})$ than that of the previous study $(30 \mathrm{mg})^{(33)}$, and the higher vitamin $\mathrm{C}$ in our MMN supplements that might enhance the absorption of Fe. Moreover, a meta-analysis also indicated that the risk of maternal anaemia in the third trimester could be decreased by an average of $34 \%$ after the supplementation with Fe-containing micronutrients during pregnancy ${ }^{(26)}$. Doseresponse analysis showed a linear decrease in maternal anaemia with higher doses of Fe, up to $66 \mathrm{mg} / \mathrm{d}^{(26)}$.
The chemical composition of Hb suggests that a deficiency of amino acids or $\mathrm{Fe}$ in the maternal diet might cause anaemia ${ }^{(34,35)}$. Increased $\mathrm{Fe}$ intake during pregnancy is necessary to meet the $\mathrm{Fe}$ requirements of the mother and the developing fetus, as well as the haemodilution, which occurs in the second and third trimesters ${ }^{(36)}$. It may suggest that the high dose of Fe in micronutrients might be a key factor. Moreover, a deficiency of vitamins (vitamin $\mathrm{B}_{6}$, vitamin $\mathrm{B}_{12}$ and folate) and minerals (cobalt, $\mathrm{Mg}, \mathrm{Zn}$ and $\mathrm{Cu}$ ) may also indirectly result in anaemia because they participate in one-carbon-unit metabolism and DNA synthesis ${ }^{(37,38)}$. Therefore, the reduction of pregnancy anaemia by the MMN supplementation might be attributed partly to such vitamins and minerals. In this sense, the MMN supplements could be a priority policy recommendation during pregnancy to prevent and control maternal anaemia.

About $70 \%$ of the women in the MMN group remained anaemic in the third trimester after the adjustment of altitudes by the CDC method. A trial in rural northern China found that prenatal Fe supplementation (300 mg ferrous sulfate) reduced anaemia, but most women still had Fe deficiency ${ }^{(39)}$. Another cluster-randomised trial conducted in rural western China found that in both Fe-FA and MMN groups more than $40 \%$ of the pregnant women were still anaemic in the third trimester ${ }^{(33)}$. Blood volume expansion during the third trimester partly accounts for the high prevalence of anaemia ${ }^{(25,40)}$. Moreover, the absorption of Fe during pregnancy is a complex physiological process, in which the Fe absorption decreases during the first trimester of pregnancy with a progressive increase in the remaining two trimesters of pregnancy because of the greater requirements for Fe in later pregnancy. However, the amounts that can be absorbed from the diet are less than the $\mathrm{Fe}$ requirements in later pregnancy ${ }^{(41)}$.

In addition, we found that although the percentage of anaemic Tibetan women during pregnancy was quite high in the present study, about half of anaemic women did not exhibit typical clinical symptoms of anaemia such as fatigue, reduced physiological endurance, shortness of breath and headache especially on exercise, paleness of skin or a rapid heartbeat ${ }^{(42-44)}$. Our previous study also found that prevalence of maternal anaemia varied with differing correction methods for altitudes $^{(6)}$, suggesting that current available methods were probably not suitable for adjusting for $\mathrm{Hb}$ level according to the altitude in pregnant women living in the highlands of Tibet. Better adaptation to high altitudes for Tibetans might account for this ${ }^{(18,19)}$, besides the unbalanced diets and poor nutritional education $^{(20)}$. 


\section{Improvement of birth outcomes}

This study pointed to the possibility that the MMN supplementation during pregnancy in Tibetan women might improve certain birth outcomes. Compared with FA, the MMN supplement group had an (albeit statistically non-significant) increase in birth weight and significantly decreased the occurrence of LBW by $42 \%$. This result implied that the MMN supplementation might positively affect Tibetan babies with LBW. We also found that the MMN supplements could extend the duration of gestation by 0.56 weeks and decreased preterm birth by $69 \%$ compared with FA. However, a significant difference was not found in the prevalence of the infant with SGA between MMN and FA $(35 \cdot 2 v \cdot 30 \cdot 1 \%$. SGA was defined as birth weight less than 10th percentile of the gestational age-sex specific US reference for fetal growth $\left.{ }^{(45)}\right)$. Thus, it was possible that MMN reduced LBW through prolonging the gestation period and thus decreasing preterm birth.

The beneficial effects of the MMN supplementation on fetal growth could be addressed through several biological mechanisms. Deficiencies of B vitamins might cause elevated homocysteine levels, which can lead to endothelial cell dysfunction and affect placental function ${ }^{(46,47)}$. Although we did not collect the data on the status of B vitamins during pregnancy in this study, our previous study found that Tibetan women with a child aged $<24$ months suffered from poor dietary intake of protein and $B$ vitamins ${ }^{(20)}$. This suggested that the MMN supplements could help maintain normal homocysteine levels during pregnancy. Deficiency of some vitamins and minerals may also bring about negative effects on gene regulation and cellular metabolism ${ }^{(46,48)}$. Moreover, the MMN supplementation could improve the nutritional and $\mathrm{Hb}$ status of Tibetan women because they require more micronutrients including vitamins and minerals during pregnancy ${ }^{(24)}$. Furthermore, MMN was found to reduce the risk of perinatal mortality by $54 \%$, which might be a clinically important finding. The reduction of perinatal mortality could be attributed to the reduction of LBW and preterm births, which was supported by evidence of the important role of antenatal Fe supplements on the reduction of LBW and preterm delivery in the previous studies ${ }^{(26,49)}$. However, these results should be interpreted cautiously because the present study was not powered to address this issue. These results, therefore, suggested that the MMN supplements might play a positive role in the improvement of birth outcomes of Tibetan babies.

The response to the micronutrient supplementation during pregnancy varied by circumstances and population. A trial in China observed that the MMN supplements containing $30 \mathrm{mg}$ of Fe significantly increased birth weight, but there was no significant reduction in LBW and early neonatal mortality. Fe-FA supplements containing $60 \mathrm{mg}$ of Fe decreased neonatal mortality, which could be attributed to high dose of $\mathrm{Fe}$ in the supplements ${ }^{(33)}$. In the present study, the MMN supplements contained $60 \mathrm{mg}$ of $\mathrm{Fe}$, and no reduction of neonatal mortality in Tibetan babies was observed, but a reduction of perinatal mortality was still observed. We thought that a smaller sample size could account for this variation, but a different physiological response to MMN for Tibetan women could not be ruled out. Similar improvement of birth outcomes by MMN supplementation was also observed in a trial in Nepal. A $77 \mathrm{~g}$ increase in average birth weight and a decrease in the incidence of LBW by $25 \%$ in the MMN group (fifteen vitamins and minerals, contained $30 \mathrm{mg}$ of $\mathrm{Fe}$ ) was observed compared with the Fe-FA group (contained $60 \mathrm{mg}$ of $\mathrm{Fe}$ ). However, no difference was recorded for the duration of gestation or infant length ${ }^{(28)}$. On the basis of the present study, the positive effect of the MMN supplementation during pregnancy on birth weight could possibly be related to the addition of Fe.

A systematic review and meta-analysis of prenatal Fe use confirmed that daily prenatal use of Fe substantially improved birth weight in a linear dose-response manner, probably leading to a reduction in the risk of LBW. However, the Fe supplementation was not related to gestational age, preterm birth and birth length ${ }^{(26)}$. As the MMN supplements for pregnant women are generally a complex of MMN with vitamins and minerals including Fe, FA and other B vitamins, other micronutrients besides $\mathrm{Fe}$ in the MMN supplements could contribute to the improvement of birth outcomes ${ }^{(24)}$. As for the effect of the MMN supplements on the mortality of babies, the results varied by study. Our previous study ${ }^{(33)}$ did not find the reduction of neonatal mortality by the MMN supplements and the present study also found that the MMN supplements did not reduce neonatal mortality other than the reduction of perinatal death. In another randomised controlled trial in northern China $^{(50)}$, the supplementation with Fe-FA (containing $30 \mathrm{mg}$ of $\mathrm{Fe}$ ) with or without other micronutrients (fifteen vitamins and minerals, contained $30 \mathrm{mg}$ of $\mathrm{Fe}$ ) did not affect the perinatal mortality rate compared with daily prenatal FA, except that relative risks for the third-trimester maternal anaemia were reduced. However, a large trial in Indonesia showed that the prenatal MMN supplementation (fifteen vitamins and minerals, contained $30 \mathrm{mg}$ of $\mathrm{Fe}$ ), as compared with Fe-FA (contained $30 \mathrm{mg}$ of $\mathrm{Fe}$ ), reduced early infant mortality (deaths until $90 \mathrm{~d}$ postpartum), especially in the undernourished and anaemic women $^{(51)}$. The results from the present study suggested that, for Tibetan women, the supplementation with Fe-containing MMN during pregnancy tended not only to reduce the prevalence of maternal anaemia but also improve birth outcomes to some extent. However, the observed effects of the MMN supplements on mortality of Tibetan babies should be interpreted with caution.

\section{Strengths and limitations}

The lack of scientific research represented a gap in the knowledge on prenatal micronutrients supplementation and its effect on Tibetan mothers and their babies, who live in the highest altitude areas in the world. The present study is the first intervention study assessing the impact of the MMN supplements during pregnancy, compared with FA, on maternal anaemia and birth outcomes in the Tibetan population. In the study, the compliance with supplementation in both arms was higher than predicted, as reflected by a mean maternal compliance rate of $88.2 \%$. To address the possible imbalance in the participants' characteristics between two treatment groups owing to the non-randomised design of this study in the 
analysis, the propensity score was established and used in the GEE model for controlling for potential confounders. Therefore, the present study provides valuable evidence for Tibetan $\mathrm{MCH}$ care policy that both Tibetan women and their babies could benefit from the MMN supplementation during pregnancy.

We recognise several limitations in the present study. First, the randomisation allocation was not performed owing to the difficulty of implementation because of cultural and religious sensitivity. Although we have tried to use the propensity score approach to control for any possible imbalance between the two groups, there still might be unobserved heterogeneities. As a result, the observed differences in the maternal anaemia and birth outcomes may be subject to possible unknown confounding factors. Second, we used FA as the control group because it was the standard prenatal supplement recommended by the Chinese Government during the period of this study. Third, the date of LMP may be inaccurate in a small part of the pregnant women and the gestational weeks were identified based on the clinical experiences of obstetricians. Consequently, the study may underestimate the preterm rate in Tibet.

\section{Conclusions}

This prospective cohort study has provided some evidence that the MMN supplements have important positive health effects for pregnant women and newborn infants, with the potential to not only reduce the prevalence of maternal anaemia during pregnancy but also improve birth outcomes to some extent in rural Tibet in high altitudes. Further randomised control trials are required to confirm such effects of this MMN formula.

\section{Acknowledgements}

The authors thank the following members of the Tibet study team that helped in the implementation of the study: ZhuomaDuoji, CangPu, Yan Xing, Xiaoyan Zhou, Leilei Pei, Chao Zhang, Guodong Wang, Chao Li and Jiangping Li. Zhenjie Wang performed computer programming and data management; GaZhuo, CuoBai and Sang Qu were responsible for computer programming and data management; and Ga Lang was the field supervisor. Yue Cheng provided useful comments about the study design and field procedures. The authors also thank the area coordinators of the Primary Health and Maternal and Child Health Division of Health Bureau of Tibet Autonomous Region, Lhasa Municipal Health Bureau and Health Bureaus in the study counties of Tibet Autonomous Region. Finally, the authors thank Emma Yu Wang for proofreading and editing the paper.

This work was supported by the China Medical Board, USA (grant no. CMB-\#02-778), and the National Natural Science Foundation of China (grant no. 30771835).

Y. K. and S. D. developed the study protocol, questionnaires and field procedures, directed the field operations, held routine meetings with community leaders and health staff, contributed to the quality control, data cleaning, data analysis, data interpretation and wrote the first draft of the paper; L. Z. contributed to the preparation of the manual of operations, and helped in training field staff and quality control; D. W. carried out the initial analyses, and reviewed and revised the manuscript; Q. L. helped in the study design, data analysis and the writing of the manuscript; J. W. and L. O. directed the field implementation, community preparation and advocacy; H. Y. was the principal investigator, designed the study hypothesis and protocol, directed the implementation of the study, supervised the field operations, data analysis and data interpretation, edited the paper and acted as the guarantor. All authors approved the final manuscript as submitted and agreed to be accountable for all aspects of the work.

The authors declare that there are no conflicts of interest.

\section{References}

1. Stevens GA, Finucane MM, De-Regil LM, et al. (2013) Global, regional, and national trends in haemoglobin concentration and prevalence of total and severe anaemia in children and pregnant and non-pregnant women for 1995-2011: a systematic analysis of population-representative data. Lancet Glob Health 1, e16-e25.

2. Black RE, Victora CG, Walker SP, et al. (2013) Maternal and child undernutrition and overweight in low-income and middle-income countries. Lancet 382, 427-451.

3. National Health and Family Planning Commission of the PRC (NHFPC) (2015) Report of nutrition and chronic disease status of Chinese residents. http://www.nhfpc.gov.cn/jkj/s5879/ 201506/4505528e65f3460fb88685081ff158a2.shtml （accessed June 2016).

4. Li M, Hu Y, Mao D, et al. (2017) Prevalence of anemia among Chinese rural residents. Nutrients $\mathbf{9}$, e192.

5. Huang L, Purvarshi G, Wang S, et al. (2015) The influence of iron-deficiency anemia during the Pregnancy on preterm birth and birth weight in South China. J Food Nutr Res 3, 570-574.

6. Xing Y, Yan H, Dang S, et al. (2009) Hemoglobin levels and anemia evaluation during pregnancy in the highlands of Tibet: a hospital-based study. BMC Public Health 9, 336.

7. Xing Y, Yan H, Dang SN, et al. (2008) [Levels of hemoglobin concentration and anemia during pregnancy in Lhasa]. Zhonghua Liu Xing Bing Xue Za Zhi 29, 668-671.

8. Kang Y, Li F, Dang S, et al. (2014) [Study on the Hemoglobin levels among the Tibetan pregnant women in rural Lhasa]. Zhonghua Yu Fang Yi Xue Za Zhi 48, 396-400.

9. Levy A, Fraser D, Katz M, et al. (2005) Maternal anemia during pregnancy is an independent risk factor for low birthweight and preterm delivery. Eur J Obstet Gynecolo Reprod Biol 122, 182-186.

10. Williams LA, Evans SF \& Newnham JP (1997) Prospective cohort study of factors influencing the relative weights of the placenta and the newborn infant. BMJ 314, 1864-1868.

11. Gonzales GF, Tapia V \& Fort AL (2012) Maternal and perinatal outcomes in second hemoglobin measurement in nonanemic women at first booking: effect of altitude of residence in Peru. ISRN Obstet Gynecol 2012, 368571.

12. Jwa SC, Fujiwara T, Yamanobe Y, et al. (2015) Changes in maternal hemoglobin during pregnancy and birth outcomes. BMC Pregnancy Childbirth 15, 80 .

13. Class QA, Rickert ME, Lichtenstein P, et al. (2014) Birth weight, physical morbidity, and mortality: a population-based sibling-comparison study. Am J Epidemiol 179, 550-558.

14. Zhou J, Zeng L, Dang S, et al. (2016) Maternal prenatal nutrition and birth outcomes on malnutrition among 7- to 10-year-old children: a 10-year follow-up. J Pediatr 178, 40-46, e43.

15. Chang S, Zeng L, Brouwer ID, et al. (2013) Effect of iron deficiency anemia in pregnancy on child mental development in rural China. Pediatrics 131, e755-e763. 
16. Zhang TL (1997) Population Development in Tibet and Related Issues, 1st ed. Beijing: Foreign Languages Press.

17. Centers for Disease Control and Prevention (1995) Altitude Hemoglobin Curve and CDC Anemia Criteria Which Uses the Altitude Adjustment. Atlanta: CDC.

18. Moore LG (1990) Maternal O2 transport and fetal growth in Colorado, Peru, and Tibet high-altitude residents. Am J Hum Biol 2, 627-637.

19. Science (2017) Mutations may reveal how Tibetans can live on world's highest plateau. http://www.sciencemag.org/news/ 2017/04/mutations-may-reveal-how-tibetans-can-live-world-shighest-plateau (accessed April 2017).

20. Wang Z, Dang S \& Yan H (2010) Nutrient intakes of rural Tibetan mothers: a cross-sectional survey. BMC Public Health 10, 801.

21. Dang S, Yan H \& Yamamoto S (2008) High altitude and early childhood growth retardation: new evidence from Tibet. Eur J Clin Nutr 62, 342-348.

22. Bianba Z, Pubu Z \& Kang YJ (2014) Analysis on the status of Tibetan newborn birth weight and its impact factors in Lhasa city. Chin J Perinat Med 17, 464-466.

23. Yu DM, Zhao LY, Liu AD, et al. (2007) [Incidence of low birth weight of neonates and the influencing factors in China]. Zhonghua Yu Fang Yi Xue Za Zhi, 41 Suppl., 150-154.

24. Haider BA \& Bhutta ZA (2015) Multiple-micronutrient supplementation for women during pregnancy. Cochrane Database Syst Rev, issue 11, CD004905.

25. Centers for Disease Control and Prevention (1998) Recommendations to prevent and control iron deficiency in the United States. Centers for Disease Control and Prevention. MMWR Recomm Rep 47, 1-29.

26. Haider BA, Olofin I, Wang M, et al. (2013) Anaemia, prenatal iron use, and risk of adverse pregnancy outcomes: systematic review and meta-analysis. BMJ 346, f3443.

27. Maternal and Child Health Division (2010) Project management plan on folic acid supplementation prevented neural tube defects. Notification of the Ministry of Health. http://www. nhfpc.gov.cn/fys/s3581/201006/942109bebb4340b2922898 f565489a6f.shtml (accessed February 2015).

28. Osrin D, Vaidya A, Shrestha Y, et al. (2005) Effects of antenatal multiple micronutrient supplementation on birthweight and gestational duration in Nepal: double-blind, randomised controlled trial. Lancet 365, 955-962.

29. McLean E, Cogswell M, Egli I, et al. (2009) Worldwide prevalence of anaemia, WHO Vitamin and Mineral Nutrition Information System, 1993-2005. Public Health Nutr 12, 444-454.

30. Steer P, Alam MA, Wadsworth J, et al. (1995) Relation between maternal haemoglobin concentration and birth weight in different ethnic groups. BMJ 310, 489-491.

31. Zeng L, Yan H \& Chen Z (2008) [Measurement of the living standards of family in rural area and relationship between wealth index and perinatal care status]. Wei Sheng Yan Jiu 37, $714-717$.

32. Yue LQ (2007) Statistical and regulatory issues with the application of propensity score analysis to nonrandomized medical device clinical studies. J Biopharm Stat 17, 1-13 discussion 15-17, 19-21, 23-17 passim.

33. Zeng L, Dibley MJ, Cheng Y, et al. (2008) Impact of micronutrient supplementation during pregnancy on birth weight, duration of gestation, and perinatal mortality in rural western China: double blind cluster randomised controlled trial. BMJ 337, a2001.

34. Wu G (2009) Amino acids: metabolism, functions, and nutrition. Amino Acids 37, 1-17.

35. Zimmermann MB (2006) The influence of iron status on iodine utilization and thyroid function. Annu Rev Nutr 26, 367-389.

36. Wu G, Imhoff-Kunsch B \& Girard AW (2012) Biological mechanisms for nutritional regulation of maternal health and fetal development. Paediatr Perinat Epidemiol, 26 Suppl. 1, $4-26$.

37. McNulty H \& Scott JM (2008) Intake and status of folate and related B-vitamins: considerations and challenges in achieving optimal status. Br J Nutr, 99 Suppl. 3, S48-S54.

38. Said HM (2011) Intestinal absorption of water-soluble vitamins in health and disease. Biochem J 437, 357-372.

39. Zhao G, Xu G, Zhou M, et al. (2015) Prenatal iron supplementation reduces maternal anemia, iron deficiency, and iron deficiency anemia in a randomized clinical trial in Rural China, but iron deficiency remains widespread in mothers and neonates. J Nutr 145, 1916-1923.

40. Scholl TO (2005) Iron status during pregnancy: setting the stage for mother and infant. Am J Clin Nutr 81, 1218S-1222S.

41. Bothwell TH (2000) Iron requirements in pregnancy and strategies to meet them. Am J Clin Nutr 72, 257S-264S.

42. Krayenbuehl PA, Naumann KU, Kaser L, et al. (2007) [Iron deficiency anemia in adults. Main symptoms: fatigue, pallor, (stress-) dyspnea, headache, concentration disorders]. Praxis (Bern 1994) 96, 1241-1247; quiz 1248.

43. Lasch KF, Evans CJ \& Schatell D (2009) A qualitative analysis of patient-reported symptoms of anemia. Nephrol Nurs $J \mathbf{3 6}$, 621-624, 631-632; quiz 633.

44. Brunner C \& Wuillemin WA (2010) [Iron deficiency and iron deficiency anemia - symptoms and therapy]. Ther Umsch $\mathbf{6 7}$, 219-223.

45. Alexander GR, Himes JH, Kaufman RB, et al. (1996) A United States national reference for fetal growth. Obstet Gynecol 87, 163-168.

46. Furness D, Fenech M, Dekker G, et al. (2013) Folate, vitamin $\mathrm{B}_{12}$, vitamin $\mathrm{B}_{6}$ and homocysteine: impact on pregnancy outcome. Matern Child Nutr 9, 155-166.

47. Qureshi SB, Ahmad M, Qureshi PMA, et al. (2010) Hyperhomocysteinaemia, vascular related pregnancy complications and the response to vitamin supplementation in pregnant women of Pakistan. J Pak Med Assoc 60, 741-745.

48. Ashworth CJ \& Antipatis C (2001) Micronutrient programming of development throughout gestation. Reproduction 122, 527-535.

49. Siega-Riz AM, Hartzema AG, Turnbull C, et al. (2006) The effects of prophylactic iron given in prenatal supplements on iron status and birth outcomes: a randomized controlled trial. Am J Obstet Gynecol 194, 512-519.

50. Liu JM, Mei Z, Ye R, et al. (2013) Micronutrient supplementation and pregnancy outcomes: double-blind randomized controlled trial in China. JAMA Intern Med 173, 276-282.

51. Shankar AH, Jahari AB, Sebayang SK, et al. (2008) Effect of maternal multiple micronutrient supplementation on fetal loss and infant death in Indonesia: a double-blind clusterrandomised trial. Lancet 371, 215-227. 